\title{
A numerical method for calculating the loss of life of power transformers
}

\begin{abstract}
Temperature, especially the hot spot temperature (HST) and top oil temperature (TOT), has played the important rule and became the most effective factor in determination of the insulation life of the transformer. The prediction of HST and TOT is very important for estimating the loss of life (LOL) of the transformer in power system. Therefore, an accurate technique is needed for solving the thermal models. This paper presents a numerical method which can accurately calculate the HST and thus provides an effective evaluation of LOL of the transformer. An alternative solution for solving the thermal model is proposed in this work and results are compared with the actual temperature, measured by fiber optic sensors and placed on the 30MV A power transformer.
\end{abstract}

Keyword: Hot spot temperature; Loss of life of the transformer; Electrical transformer 